\title{
An Analytical Prediction Model of Time Diversity Performance for Earth-Space Fade Mitigation
}

\author{
Pantelis-Daniel M. Arapoglou, Athanasios D. Panagopoulos, and Panayotis G. Cottis \\ Wireless \& Satellite Communications Group, Division of Information Transmission Systems and Materials Technology, School of \\ Electrical and Computer Engineering, National Technical University of Athens, Heroon Polytechniou 9, 15780 Zografou, Greece \\ Correspondence should be addressed to Athanasios D. Panagopoulos, thpanag@cc.ece.ntua.gr
}

Received 14 August 2007; Accepted 25 January 2008

Recommended by Krishnasamy Selvan

Time diversity (TD) has recently attracted attention as a promising and cost-efficient solution for high-frequency broadcast satellite applications. The present work proposes a general prediction model for the application of TD by approximating the time dynamics of rain attenuation through the use of the joint lognormal distribution. The proposed method is tested against experimental data and its performance is investigated with respect to the basic parameters of a satellite link.

Copyright (c) 2008 Pantelis-Daniel M. Arapoglou et al. This is an open access article distributed under the Creative Commons Attribution License, which permits unrestricted use, distribution, and reproduction in any medium, provided the original work is properly cited.

\section{INTRODUCTION}

Several diversity reception schemes have been thoroughly investigated in the past to mitigate rain fading in fixed/broadcast satellite communication systems operating above $10 \mathrm{GHz}$. These schemes offer a significant performance improvement, especially considering the recent trend of commercial satellite networks to migrate to higher-frequency bands due to spectral and/or orbital congestion: first, from $\mathrm{C}$ to the $\mathrm{Ku}$ frequency band and, more recently, from $\mathrm{Ku}$ to the Ka, or even to the EHF frequency band. The established diversity techniques include [1]

(i) site diversity (SD), which takes advantage of the spatial characteristics of the rainfall medium by engaging two or more jointly receiving earth terminals to combat strong rain fades [2];

(ii) orbital (or satellite) diversity (OD), which takes advantage of the spatial characteristics of the rainfall medium by allowing earth stations to choose the best signal from two or more satellites [3];

(iii) frequency diversity (FD), which takes advantage of the spectral characteristics of the rainfall medium by employing a high-frequency band (e.g., Ka or EHF) during normal operation, and switching to a lowerfrequency band (e.g., Ku or C) when the attenuation due to rain exceeds a certain threshold [4].
All the above fade mitigation techniques (FMT) produce significant diversity gains but their use is limited due to specific technical and other factors. For SD, the main limitation comes from cost considerations, since additional earth stations and terrestrial connections enabling the processing of the jointly received signals are required. In OD, limitations are imposed by the switching procedure between the satellites, as well as by the waste of satellite bandwidth. Finally, FD is rather expensive, requiring dual reception at the earth terminals.

Time Diversity (TD), is a less studied diversity alternative for satellite systems [5]. Basically, it consists of retransmitting the corrupted information at times when the channel is expected to be more favourable, that is at time spacings exceeding the channel coherence time. The basic principle of the TD technique is depicted in Figure 1. Actually, it resembles the ARQ (automatic repeat request) FMT implemented in layer 2, which falls under the general category of error correction. The difference between the two techniques is that ARQ is characterized by a fixed or random retransmission period, while TD retransmits the information after having estimated the duration of the unfavorable propagation phenomenon. Clearly, the performance of the TD technique is closely related to the time period selected for retransmission, which ranges from a few seconds to several hours. One of its main advantages when compared to other diversity 


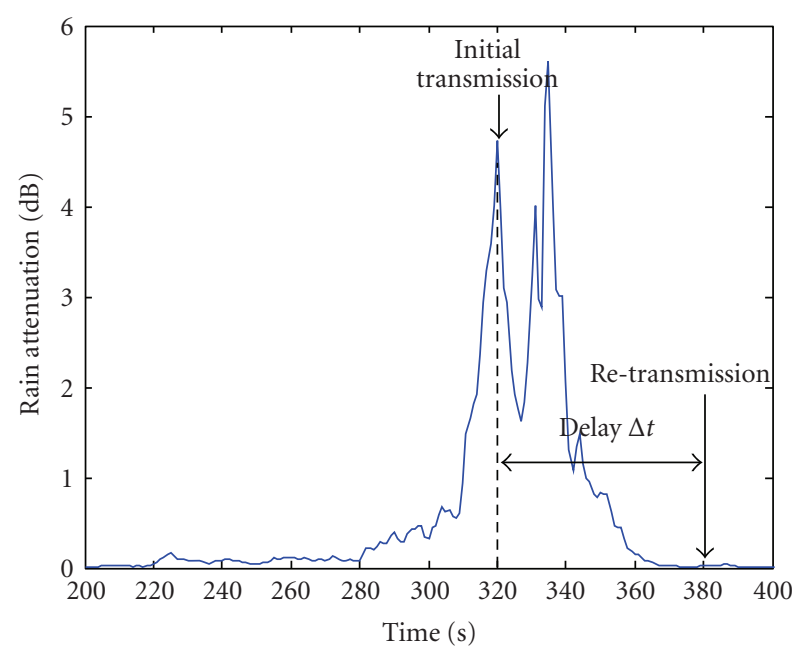

Figure 1: Application of the TD technique.

techniques is that TD does not require additional equipment or complicated synchronization procedures, since it involves only a single satellite link and a single reception unit. This renders the TD option cost efficient. However, the application of the TD technique is restricted to delay-tolerant applications, such as video-on-demand or multimedia and data transfer, and seems particularly attractive for broadcasting services.

Given that only a few similar models exist in the open literature (briefly outlined in Section 2), usually derived from measurements in specific regions, the present paper proposes a physical prediction model for the performance estimation of TD. The model, detailed in Section 3, relies on the assumption that the joint statistics of rain attenuation at both the initial and the retransmission instants follow the lognormal distribution. In Section 4, the prediction model is tested against TD experimental data and the main parameters influencing its performance are discussed.

\section{RELATED MEASUREMENT AND MODELING ACTIVITIES}

One of the first TD measurement campaigns was carried out for the equatorial region of Malaysia using a satellite beacon receiver at $12 \mathrm{GHz}$ to record data from a MEASAT geostationary satellite. Apart from presenting the analysis of the 20month data, an empirical exponential model to fit the cumulative distribution of TD was developed [6]. This distribution is defined by means of the exceedance probability

$$
\operatorname{Pr}\left\{A_{\mathrm{TD}}(\Delta t)>a\right\}=1-\operatorname{Pr}\{\min (A(t), A(t+\Delta t)) \leq a\},
$$

where $A(t)$ is the sample of the rain attenuation stochastic process at time $t, A_{\mathrm{TD}}(\Delta t)$ denotes the attenuation that a TD system with a retransmission time of $\Delta t$ is subjected to, and $a$ is the fade margin. All attenuation quantities are expressed in $\mathrm{dB}$. Furthermore, TD measurements from the South of England in the V frequencies bawd were reported in [7] using ITALSAT beacon data. The duration of this measurement campaign has been three years. Valuable insight on TD both through experimental data and empirical modeling for the area of Japan is given in [8], which includes eight years of attenuation data at $20 \mathrm{GHz}$. The relationship between TD gain, time delay, and time percentage was approximated by a function similar to the one proposed by the ITU-R Recommendation [9, page 618] for single site rain attenuation prediction. Note that the TD gain for the $p \%$ time percentage is given by the difference

$$
G_{\mathrm{TD}}(\Delta t ; p \%)=A_{\mathrm{TD}}(\Delta t=0 ; p \%)-A_{\mathrm{TD}}(\Delta t \neq 0 ; p \%) .
$$

Results of the data processing for TD from the ITALSAT geostationary satellite were presented in [10]. The data were collected at $19 \mathrm{GHz}$ in Italy and at 12, 20, 30, 40, and $50 \mathrm{GHz}$ in Belgium over an observation period of six years. The authors in [10] assume the bivariate lognormal distribution to model the joint statistics of two attenuation random variables $A(t)$, $A(t+\Delta t)$ separated by a time delay of $\Delta t$. The correlation between these random variables in the time domain is assumed to follow the same distribution as the corresponding spatial correlation function employed in SD. This is taken into account by simply replacing the site separation distance with the TD delay. Finally in [11], a statistical assessment of TD is presented by simulating rain attenuation time series from rain rate data with the synthetic storm technique (SST) in the frequency range $10-100 \mathrm{GHz}$. The rain rate databank spans over a period of seven years.

\section{PROPOSED TD MODEL}

To simplify the notation, the rain attenuation random variables corresponding to the first and the second transmission of the same signal are here after denoted by $A_{1}$ and $A_{2}$, respectively. The starting point of the proposed approach is the assumption that the two samples $A_{1}=A(t)$ and $A_{2}=$ $A(t+\Delta t)$ of the rain attenuation random process follow the lognormal distribution. Based on this hypothesis, it can easily be shown that the exceedance probability given by (1) may also be determined by

$$
P_{\mathrm{TD}}=\operatorname{Pr}\left(A_{1}>a, A_{2}>a\right)=\iint_{a}^{\infty} p_{A_{1} A_{2}}\left(A_{1}, A_{2}\right) d A_{1} d A_{2},
$$

where $p_{A_{1} A_{2}}\left(A_{1}, A_{2}\right)$ is the joint probability density function (pdf) of $A(t)$ and $A(t+\Delta t)$. The above joint pdf is assumed to follow the bivariate lognormal distribution. The exceedance probability of a single transmission is (setting for simplicity $A=A(t))$

$$
\operatorname{Pr}\left(A_{1}>a\right)=\int_{a}^{\infty} p_{A}(A) d A=\frac{1}{2} \operatorname{erfc}\left(\frac{\ln a-\ln A_{m}}{\sqrt{2} S_{a}}\right),
$$

where $p_{A}(A)$ is the lognormal pdf, $A_{m}$ and $S_{a}$ are the mean value and the standard deviation of the corresponding normal random variable $\ln A$, and $\operatorname{erfc}(x)$ is the complementary error function (4) [3]. Based on the transformation

$$
U=\frac{\ln A-\ln A_{m}}{S_{a}}
$$


the exceedance probability given by (4) is expressed as a function of the normalized attenuation $U$

$$
\operatorname{Pr}(U>u)=\frac{1}{2} \operatorname{erfc}\left(\frac{u}{\sqrt{2}}\right) .
$$

Assuming that long-term rain attenuation measurements are available in the form of attenuation probability pairs, $\left(A_{i}, P_{i}\right), i=1, \ldots, N$, where $N$ is the number of available data pairs, the statistical parameters $A_{m}$ and $S_{a}$ may be evaluated by first reversing (6) to obtain

$$
U_{i}=\sqrt{2} \operatorname{erfc}^{-1}\left(2 P_{i}\right), \quad i=1, \ldots, N .
$$

Since $U_{i}$ also satisfy (5), the following set of equations is obtained:

$$
U_{i}=C_{1} Z_{i}+C_{2}, \quad i=1, \ldots, N,
$$

where

$$
\begin{aligned}
Z_{i} & =\ln A_{i}, \\
C_{1} & =S_{a}^{-1}, \\
C_{2} & =-\ln A_{m} / S_{a} .
\end{aligned}
$$

Since $U_{i}$ and $Z_{i}$ are related through the linear relationship (8), a least-squares regression procedure may easily lead to the evaluation of the coefficients $C_{1}$ and $C_{2}$ and, then, to $A_{m}$ and $S_{a}$. Since to obtain (4) and (6) the unconditional lognormal distribution is adopted, in the calculations only values of $A_{i}$ higher than a minimum $0.5 \mathrm{~dB}$ threshold are taken into account. Also, in case local measurements are not available, the statistical parameters $A_{m}$ and $S_{a}$ may be calculated applying the procedure, summarized in (6)-(9), to the rain attenuation model of the ITU-R Recommendation [9, page 618].

Returning to the bivariate case and adopting for both random variables $A_{1}$ and $A_{2}$ the transformation (5), two normal random variables come up, namely, $U_{1}$ and $U_{2}$, each with zero mean and standard deviation equal to 1 . Their joint pdf is given by

$$
\begin{aligned}
& P_{U_{1} U_{2}}\left(U_{1}, U_{2}\right) \\
& =\frac{1}{2 \pi \sqrt{1-\rho_{n}^{2}(\Delta t)}} \exp \left\{-\frac{1}{2\left(1-\rho_{n}^{2}(\Delta t)\right)}\right. \\
& \left.\cdot\left(U_{1}^{2}+U_{2}^{2}-2 \rho_{n}(\Delta t) U_{1} U_{2}\right)\right\},
\end{aligned}
$$

where the normalized time correlation coefficient $\rho_{n}$ is defined by $[12,13]$

$$
\rho_{n}(\Delta t)=\frac{\exp \left(S_{a}^{2} \exp \left(-d_{A}|\Delta t|\right)\right)-1}{\exp \left(S_{a}^{2}\right)-1} .
$$

It should be emphasized at this point that the critical dependence of $p_{U_{1} U_{2}}\left(U_{1}, U_{2}\right)$ and of the subsequent results on $\Delta t$ is expressed by the correlation coefficient, which is a function of $\Delta t$.

The parameter $d_{A}\left[\mathrm{~s}^{-1}\right]$ is the dynamic parameter of rain attenuation (also referred to as beta in the literature) describing the temporal variation of the rain attenuation process. A comprehensive investigation on how to calculate $d_{A}$ based on electrical, geometrical, and climatic characteristics of the link is carried out in [14].

Based on Bayes' theorem, (10) may be expressed in terms of its marginal and conditional pdfs

$$
p_{U_{1} U_{2}}\left(U_{1}, U_{2}\right)=p_{U_{2}}\left(U_{2}\right) \cdot p_{U_{1} \mid U_{2}}\left(U_{1}, U_{2}\right)
$$

where

$$
\begin{aligned}
& p_{U_{1} \mid U_{2}}\left(U_{1}, U_{2}\right) \\
& =\frac{1}{\sqrt{2 \pi\left(1-\rho_{n}^{2}(\Delta t)\right)}} \exp \left\{-\frac{\left(U_{1}-\rho_{n}(\Delta t) \cdot U_{2}\right)^{2}}{2\left(1-\rho_{n}^{2}(\Delta t)\right)}\right\},
\end{aligned}
$$

and $p_{U_{2}}\left(U_{2}\right)$ is the normal pdf. Upon replacing (12), (13) into (3), the final expression for the joint exceedance probability of the two rain attenuation random variables separated in time by $\Delta t$ comes up

$$
P_{\mathrm{TD}}=\frac{1}{2} \int_{u}^{\infty} p_{U_{2}}(U) \operatorname{erfc}\left(\frac{u-\rho_{n}(\Delta t) U}{\sqrt{2\left(1-\rho_{n}^{2}(\Delta t)\right)}}\right) d U .
$$

\section{PERFORMANCE EVALUATION}

Next, a first attempt to validate the TD statistical model based on the bivariate lognormal distribution is carried out for a variety of measured data related to various climatic zones and for links of various electrical and geometrical characteristics. The measured results for TD are simply reproduced from the open literature, as briefly outlined in Section 2.

The statistical parameters of the lognormal distribution $A_{m}$ and $S_{a}$ required for the application of the proposed model are obtained by fitting the experimental data of the exceedance probability concerning the single transmission. The dynamic parameter $d_{A}$ is determined following the methology presented in [14]. The parameters employed in the following figures are given in Table 1.

In Figure 2, TD curves for data originating from measurements reported in [7] for Sparsholt, UK, together with curves obtained applying the proposed model are depicted. A very good convergence is observed between the two sets of curves demonstrating the validity of the proposed model.

In Figure 3, the results of the proposed model are compared to single transmission TD experimental results from Kuala Lumpur, MLA [6]. The deviation observed in Figure 3 between the appropriately modified lognormal pdf and the curves depicting the measurement is mainly due to the failure of the lognormal model to represent rain attenuation in subtropical and tropical regions of the Earth, where the gamma distribution exhibits an improved prediction performance [15].

On the other hand, the overall performance of the proposed TD model depends critically on the value selected for the dynamic parameter $d_{A}$. Although in Figures 2 and 3 the evaluation of $d_{A}$ was based on the analytical methodology described in [14], there are several suggestions for a global 
TABle 1: Parameters employed.

\begin{tabular}{lccccc}
\hline Experiment & Frequency of operation to & Elevation angle & $A_{m}$ & $S_{a}$ & $d_{A}$ \\
\hline Sparsholt, UK & $49.5 \mathrm{GHz}$ & $29.9^{\circ}$ & 1.1845 & 0.9359 & $2.1 \cdot 10^{-4} \mathrm{~s}^{-1}$ \\
Kuala Lumpur, MLA & $12 \mathrm{GHz}$ & $77.43^{\circ}$ & 0.03219 & 1.8791 & $1.4 \cdot 10^{-4} \mathrm{~s}^{-1}$ \\
Spino d' Adda & $19 \mathrm{GHz}$ & $30.6^{\circ}$ & 0.11456 & 1.3352 & $1.0 \cdot 10^{-4} \mathrm{~s}^{-1}$ \\
\hline
\end{tabular}

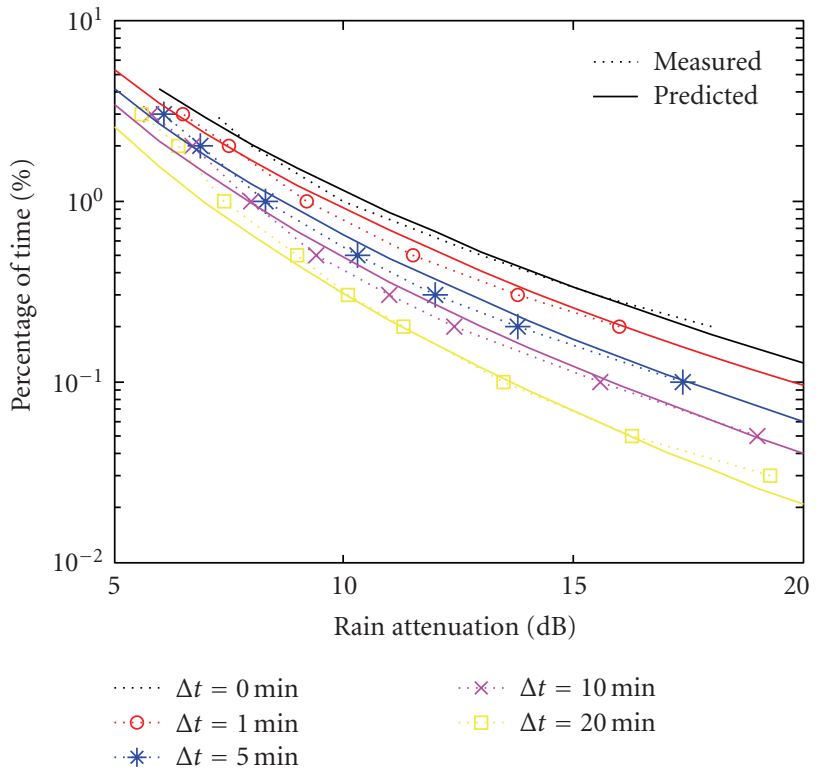

Figure 2: Performance comparison of the proposed model and measured data from a TD system operating at $50 \mathrm{GHz}$ in Sparsholt, UK.

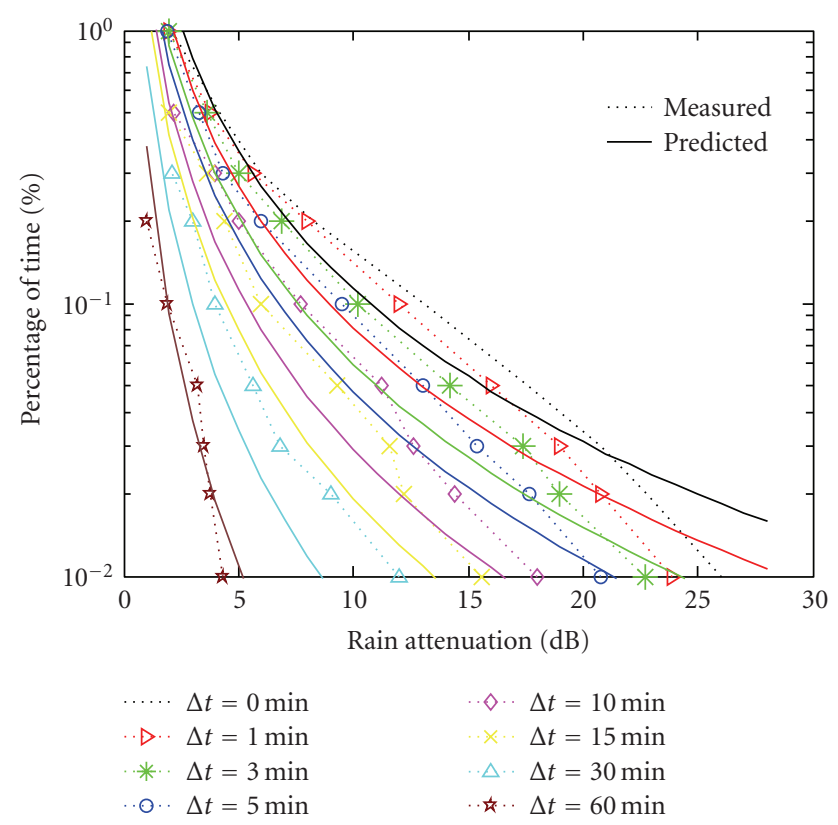

Figure 3: Performance comparison of the proposed model and measured data from a TD system operating at $12 \mathrm{GHz}$ in Kuala Lumpur, MLA.

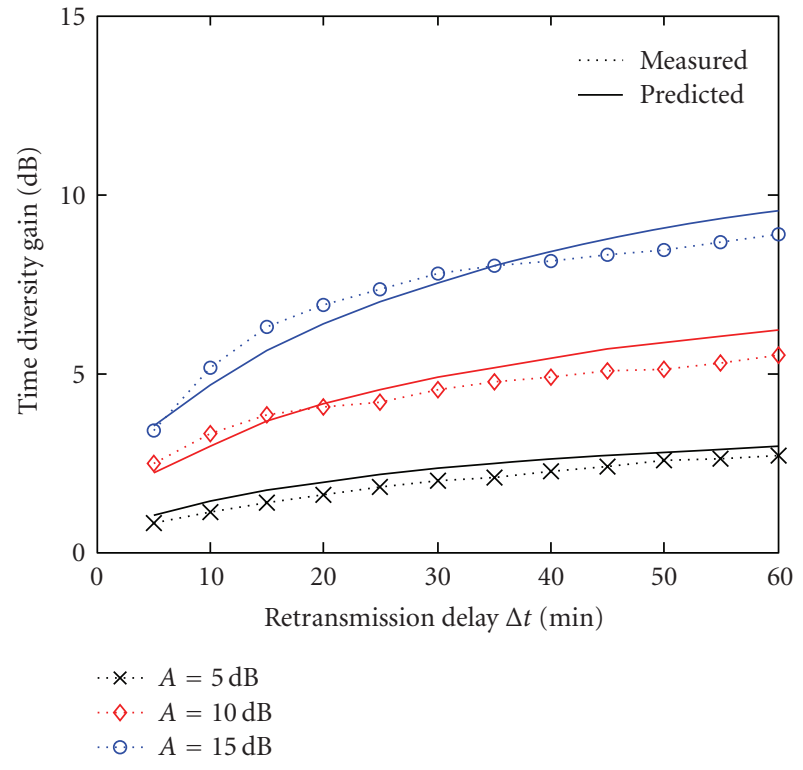

FIgure 4: Proposed model and measured data in terms of time diversity gain for a TD system operating at $19 \mathrm{GHz}$ in Spino d' Adda, IT.

value of this parameter in the range $3.16 \cdot 10^{-4} \mathrm{~s}^{-1} \leq d_{A} \leq$ $3.16 \cdot 10^{-3} \mathrm{~s}^{-1}$. Furthermore, after testing experimental data from the OLYMPUS and ITALSAT campaigns, the authors in [16] suggest that a rough estimate of $d_{A}=10^{-4} \mathrm{~s}^{-1}$ can be used in North western European regions for elevation angles between $25^{\circ}$ and $38^{\circ}$ and frequencies between $12 \mathrm{GHz}$ and $50 \mathrm{GHz}$. This value has been adopted to determine the TD gain as a function of the retransmission delay $\Delta t$ in the area of Spino d' Adda, IT. TD gain curves adopting and applying the proposed model have been plotted in Figure 4. A very satisfactory convergence between the experimental values and those of the proposed model is observed, demonstrating the validity of the proposed model.

\section{CONCLUSIONS}

The present paper proposes a general TD prediction method based on the joint correlated bivariate lognormal distribution in the time domain. The correlation function proposed depends critically on the dynamic parameter of rain attenuation. The proposed model exhibits a very satisfactory convergence to experimental data originating from temperate climatic regions. 


\section{REFERENCES}

[1] A. D. Panagopoulos, P.-D. M. Arapoglou, and P. G. Cottis, "Satellite communications at $\mathrm{Ku}, \mathrm{Ka}$, and $\mathrm{V}$ bands: propagation impairments and mitigation techniques," IEEE Communications Surveys and Tutorials, vol. 6, no. 3, pp. 2-14, 2004.

[2] A. D. Panagopoulos, P.-D. M. Arapoglou, G. E. Chatzarakis, J. D. Kanellopoulos, and P. G. Cottis, "Unbalanced large scale multiple site diversity performance in satellite communication networks," in Proceedings of the 28th URSI General Assembly, New Delhi, India, October 2005.

[3] A. D. Panagopoulos and J. D. Kanellopoulos, "A simple model for orbital diversity gain on earth-space propagation paths," IEEE Transactions on Antennas and Propagation, vol. 51, no. 6, pp. 1403-1405, 2003.

[4] L. Dossi, G. Tartara, and E. Matricciani, "Frequency diversity in millimeter wave satellite communications," IEEE Transactions on Aerospace and Electronic Systems, vol. 28, no. 2, pp. 567-573, 1992.

[5] H. Fukuchi, "Slant path attenuation analysis at $20 \mathrm{GHz}$ for time-diversity reception of future satellite broadcasting," in Proceedings of the URSI-F Open Symposium Colloque, pp. 6.5.1-6.5.4, Ravenscar, UK, 1992.

[6] A. F. Ismail and P. A. Watson, "Characteristics of fading and fade countermeasures on a satellite-Earth link operating in an equatorial climate, with reference to broadcast applications," IEE Proceedings: Microwaves, Antennas and Propagation, vol. 147, no. 5, pp. 369-373, 2000.

[7] S. Ventouras, C. L. Wrench, and S. A. Callaghan, "Measurement and analysis of satellite beacon transmissions at frequencies up to $50 \mathrm{GHz}$. Part 2: fade \& inter-fade duration," RCRU Project Report D40-3, 2001.

[8] H. Fukuchi and T. Nakayama, "Quantitative evaluation of time diversity as a novel attenuation mitigation technology for future high speed satellite communication," IEICE Transactions on Communications, vol. 87, no. 8, pp. 2119-2123, 2004.

[9] ITU-R, "Propagation data and prediction methods required for the design of Earth-space telecommunication systems," Propagation in Non-Ionized Media, Rec. P.618-8, Geneva, 2003.

[10] V. Fabbro and L. Castanet, "Characterisation and modelling of time diversity in 20-50 GHz band," in Proceedings of the European Conference on Antennas and Propagation (EuCAP '06), Nice, France, November 2006.

[11] E. Matricciani, "Time diversity as a rain attenuation countermeasure in satellite links in the 10-100 GHz frequency bands," in Proceedings of the European Conference on Antennas and Propagation (EuCAP '06), vol. 626 SP, Nice, France, November 2006.

[12] B. C. Gremont and D. L. Ndzi, "First-order and conditional statistics of rain attenuation fade slope," Electronics Letters, vol. 36, no. 20, pp. 1733-1734, 2000.

[13] T. Maseng and P. M. Bakken, "A stochastic dynamic model of rain attenuation," IEEE Transactions on Communications Systems, vol. 29, no. 5, pp. 660-669, 1981.

[14] A. D. Panagopoulos and J. D. Kanellopoulos, "On the rain attenuation dynamics: spatial-temporal analysis of rainfall rate and fade duration statistics," International Journal of Satellite Communications and Networking, vol. 21, no. 6, pp. 595-611, 2003.

[15] P.-D. M. Arapoglou, E. Kartsakli, G. E. Chatzarakis, and P. G. Cottis, "Cell-site diversity performance of LMDS systems op- erating in heavy rain climatic regions," International Journal of Infrared and Millimeter Waves, vol. 25, no. 9, pp. 1345-1359, 2004.

[16] F. Lacoste, M. Bousquet, L. Castanet, F. Cornet, and J. Lemorton, "Improvement of the ONERA-CNES rain attenuation time series synthesizer and validation of the dynamic characteristics of the generated Fade events," Space Communications, vol. 20, no. 1-2, pp. 45-59, 2005. 

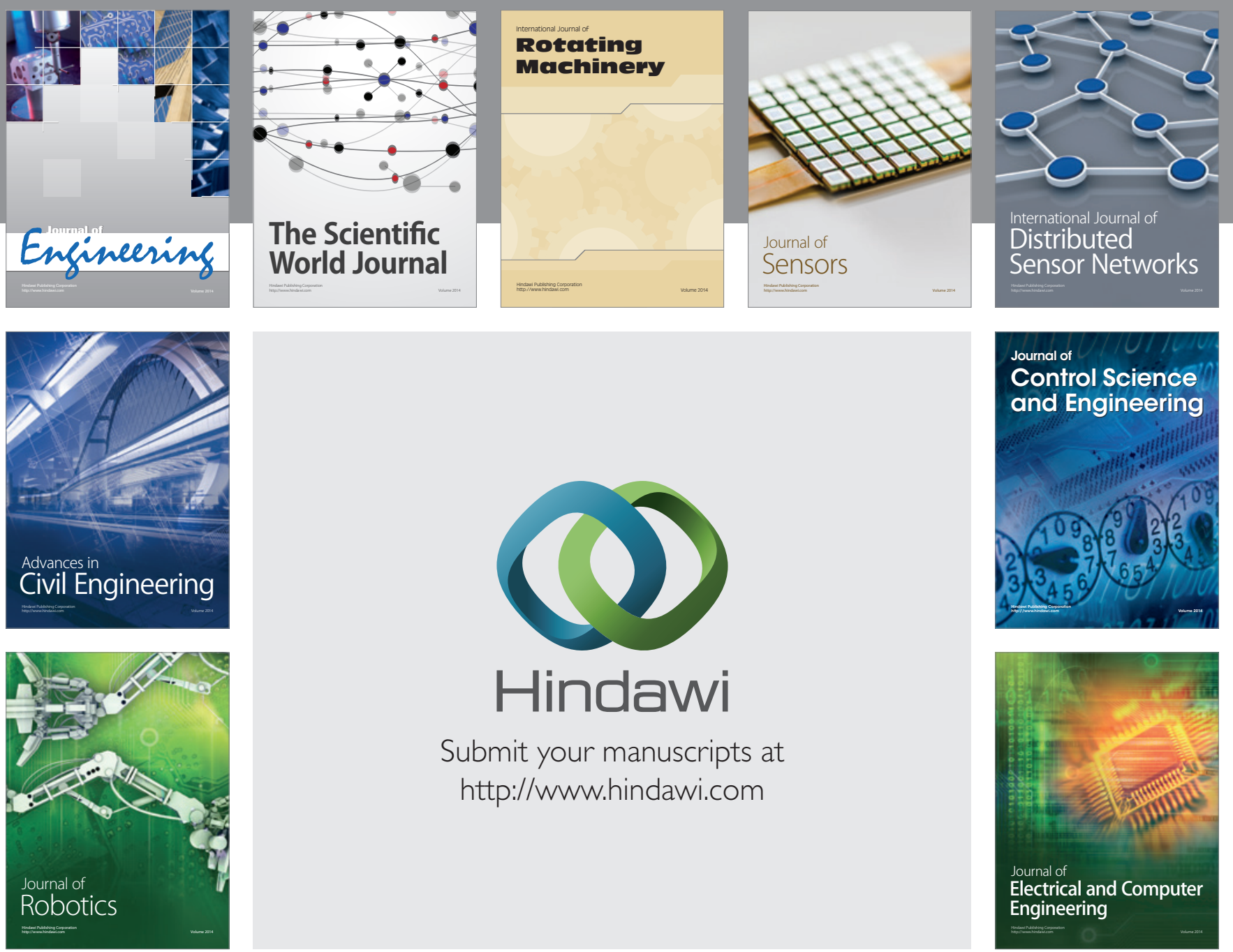

Submit your manuscripts at

http://www.hindawi.com
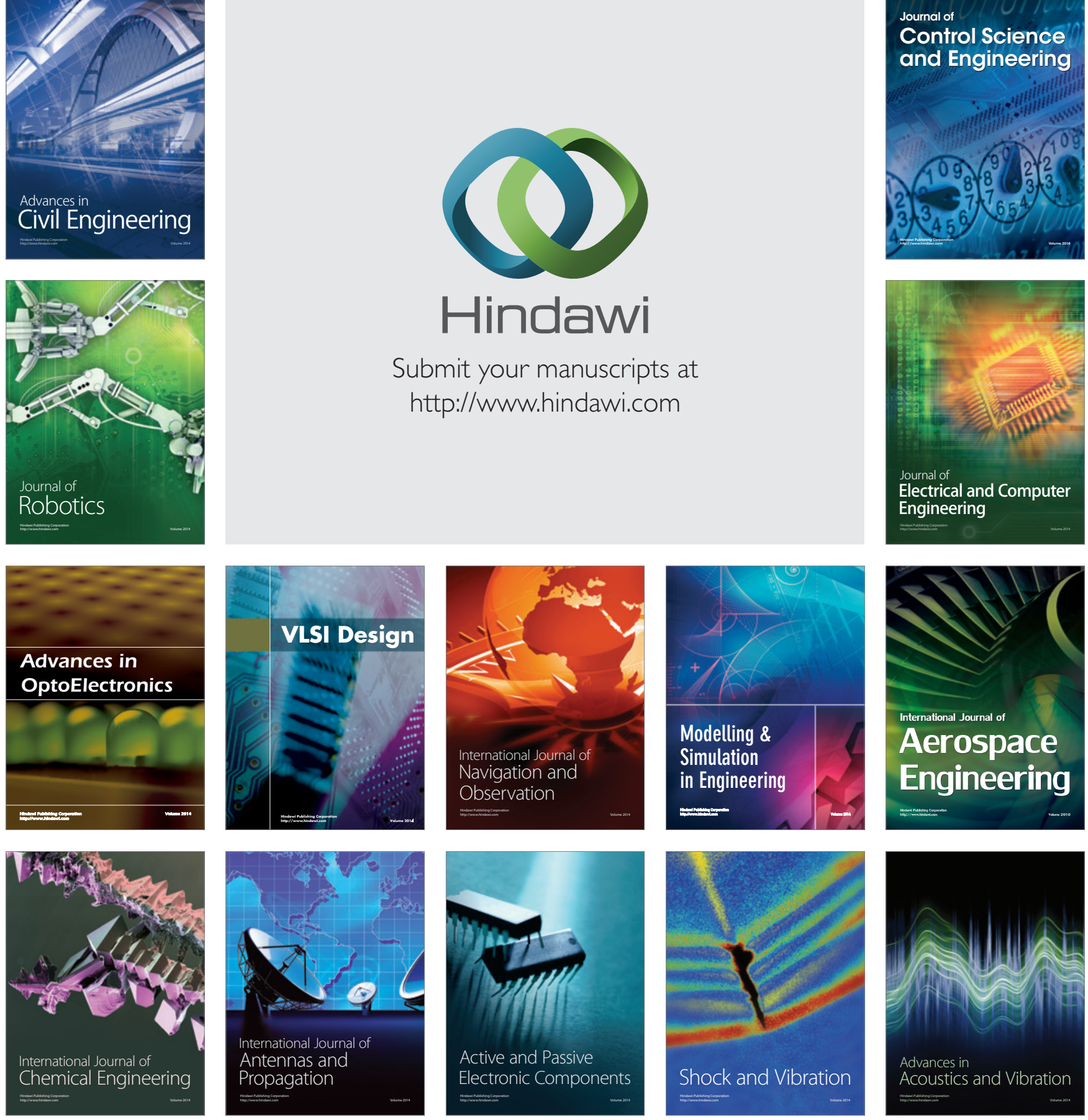